\title{
Optimization, niche and neutral mechanisms in the formation of biodiversity
}

\author{
Bukvareva Elena ${ }^{1,}$, Aleshchenko Gleb ${ }^{2}$ \\ ${ }^{1}$ Severtsov Institute of Ecology and Evolution Russian Academy of Sciences, Moscow, Russia \\ ${ }^{2}$ Lomonosov Moscow State University, the Department of Geography, Moscow, Russia
}

\section{Email address:}

bukvareva@gmail.com (Bukvareva Elena)

\section{To cite this article:}

Bukvareva Elena, Aleshchenko Gleb. Optimization, Niche and Neutral Mechanisms in the Formation of Biodiversity. American Journal of Life Sciences Vol. 1, No. 4, 2013, pp. 174-183. doi: 10.11648/j.ajls.20130104.16

\begin{abstract}
Today the main scientific belief on the mechanisms for the formation of community structure is the concept of ecological niches and the "neutral theory". We consider processes of diversity optimization at the levels of populations and ecological communities as an additional type of the mechanisms. Basing on the principle of optimal diversity a scheme for cooperative effect of different mechanisms of formation of the communities species structure is proposed: $1 \square$ number of species and their niches width is primarily determined by the processes of diversity optimization, according with an available resources levels and the degree of environmental stability; 2 - these parameters are modified by other mechanisms during the formation of the species composition, depending on the ratio of the environmental "richness" and stability: $\square$ neutral mechanisms work primarily in very "rich" or very stable environments; $\square$ niche mechanisms work primarily in the environments with medium values of the "richness" and stability, rather when the number of niches with optimal width, which divide the available range of the resource parameter and the number of species that may exist on the available quantity of the resource, are approximately equal; $\square$ "abiotic filters" work primarily in poor, severe, or extremely unstable environments.
\end{abstract}

Keywords: Biological Diversity, Phenotypic and Species Diversity, Optimization, Ecological Niches

\section{Introduction}

Identifying the mechanisms, forming species diversity and community structure, is traditionally one of the main areas of environmental research. Today it is particularly important, given the crucial role of biodiversity in providing of vital ecosystem functions to humans [1].

The basic concept to explain the mechanisms of community formation is the theory of ecological niches. It remained one of the central themes of ecological studies for decades, although recently there has been some decline of interest in this concept [2] together with the increasing popularity of "neutral theory". Another possible type of mechanisms of community structure formation is an optimization of intra-species and intrapopulation diversity $[3,4]$. The purpose of this publication is an analysis of the possible relationships between these ways of forming of the ecological community structure.

\section{Niche and Neutral Mechanisms of Species Coexistence}

\subsection{Competitive Exclusion - the Rule or the Exception to the Rule}

The idea that species which use the same resources and need the same conditions, can't live together, exists more than 100 years [5]. Following the works of V. Volterra, and G Gause in 1920-30, the idea that species can't coexist stably in one ecological niche was accepted as one of the major ecological laws and has been called "the competitive exclusion principle" or "Volterra-Gause principle". The very formulation of this principle contains a mechanism for coexistence of species - it is separation of their ecological niches.

Niche mechanisms determine the number of species as a result of "packaging" of species niches in the community. The "rule of limiting similarity" answers the question, what is the maximum degree of niches overlap, which allows species to coexist on a one resource. It has been shown 40 
years ago for the modifications of Lotka-Volterra model that the probability of species coexistence is large enough, if the mean values of the functions of the resources consuming differ no less than the standard deviation of these functions [6]. Another formulation suggests that the stronger the species niches overlap is, the lower is the range of their coexistence conditions [7]. Since this rule has not fundamentally changed, despite the development of the much more sophisticated models, e.g. [8]. The "rule of diversity of limiting factors" considers the division of multi-dimensional niche space by species. The simplest variant of the species coexistence is the use of different resources, when the niches do not overlap. In a more complex case all of the species use the same range of resources, but these species differ one from another by limiting resources $[9,10]$.

Investigations of competition have been the subject of a great amount of experiments, observations in nature and theoretical models which confirm that the competition and the ecological niches separation do exist. But these facts of the existence of species in different niches do not refute the possibility of coexistence of species in a single niche.

The principle of competitive exclusion is contrary to the diversity of wildlife that exists. The question was posed in the most vivid form by the example of the G. Hutchinson plankton community, where dozens of species of microscopic algae and cyanobacteria live together on the surface layer of water, competing for a very limited set of resources $\square$ the light, some mineral nutrients and carbon dioxide. The same question was posed in respect of a huge variety of tropical forests and coral reefs [11]. In recent years, studies confirm the possibility of different species niches broad overlapping. Thus, it has been shown for tropical and deciduous forests, that the interspecific variability of different species of trees is higher than the intraspecific one $[12,13]$. For example, average growth rates of different trees species in the southeast USA have shown almost equal response to inter-annual changes in moisture content, moreover, each species has been revealed to have a broad variability of individual trees responses, far exceeding the average differences between species [13]. Almost complete overlap of niches was found in the grass and moor frogs in different parts of their habitat [14]. Broad overlapping of niches is also determined for invasive species. For example, there are two species of Elodea, penetrated into Europe, with very similar ecological requirements and the wide overlap of niches for a growing period [15].

Of particular interest are sibling species which are found in different taxa of animals and in different geographical regions $\square$ from the pole $\square$ to the equator $[16,17]$. In many cases after the identification of sibling species former species-generalists appeared to be a complex of a few species-specialists. First of all, it concerns the cases of herbivorous insects specialization on different plant species $[18,19,20]$ or specialization of insect parasitoids in different insect species larvae [21, 22], that is determined by the species diversity of the hosts. However, some sibling species have only minor environmental differences or have almost identical environmental requirements, for example, wasps which pollinate one fig species [23].

\subsection{Mechanisms to Get Around the Principle of Competitive Exclusion}

The search for mechanisms to evade the competitive exclusion and provide coexisting of species has formed a vast area of research. There were proposed more than a hundred of mechanisms (e.g. negative density-dependent regulation of population size, the mutual suppression of species, circular competitive networks, fluctuations in environmental conditions, cyclic successions, spotted environment, varying properties of species in the space, influence of predators and parasites on competing species, mutualistic relationships with other species, chaotic regimes population dynamics, etc.) and a few alternative classifications of them $[5,24,25,26,27,28]$.

The "neutral theory" may be considered the most radical way to get around the principle of competitive exclusion. The accumulation of data on the coexistence of ecologically similar species has led to the idea that species can coexist not because of their differences, but because of their similarity. "Neutral theory" [29, 30, 31] developed rapidly and was the concept most frequently modeled and tested in environment $[32,33,34]$. Under the concept of neutral theory the local community is a part of the surrounding meta-community which species migrates constantly to the local one by analogy with the concept of equilibrium in the MacArthur-Wilson theory of island biogeography. Neutral mechanisms explain the number of species in the community as a result of the rates of migration, fertility and mortality balance in different species. Some models take also into account the rate of species speciation and extinction. Species composition may vary at that $\square$ a random process of species replacement ("ecological drift") exists.

The "neutral theory" explains the distribution of the species abundance better than the niche theory for some communities [35, 36, 37], but worse for others [38]. However, the recent models shows that the character of the distributions can't unambiguously point to the character of the mechanisms as similar distributions of species diversity can be obtained both in neutral and niche models [28, 39, 40].

\subsection{The "Niche-Neutral" Continuum of Species Coexistence}

In fact, niche and neutral theories do not contradict each other.

Competition models based on the Lotka-Volterra equations show that the coexistence of species may result from differences between them as well as from their similarities. Exploring these possibilities, P. Chesson [25] has marked out two main types of mechanisms of species 
coexistence: equalizing (neutral) mechanisms that minimize the differences between species fitness; stabilizing (niche) mechanisms that increase intraspecific competition with increasing of dominance of any species, which leads to a decrease in the rate of its growth. Separately, either equalizing or stabilizing mechanisms can't provide the stable coexistence of species. The coexistence is most probable if there are certain combinations of the mechanisms [25, 39]. Actually, the both types of mechanisms are likely to act [39, 41, 42], which is confirmed by empirical tests [35]. That is, there is a continuum of conditions, where various combinations of alignment and stabilization are between the extremes. Depending on the predominance of certain mechanisms, researchers have marked out two types of communities: a) formed primarily by mechanisms of niche separation, and b) formed primarily by neutral mechanisms of dispersal of organisms.

One of the approaches, combining neutral and niche mechanisms, worth mentioning, is the concept of "emerging groups" [42], implying that there are groups of species in the community that are sufficiently close in their ecology for neutral behavior, have similar functional niches and convergent environmental strategies. Neutral mechanisms act primarily within these groups and the niche mechanisms - between them. Interestingly, in one of the models competing species are not evenly distributed on the axis of resource but, contrary to the expectation of the authors, are distributed by groups, and the greater the width of the species niches within the groups, the greater the distance between the groups is [43].

\section{The Principle of Optimal Diversity as an Additional Mechanism for the Diversity Formation}

Thus, there are a lot of empirical data of the both competition and niche separation on the one hand, and of the broad overlap of niches and species coexistence in the nearly identical niches on the other hand. It is proposed a broad range of mechanisms to provide an implementation of these states. But the question remains why there are as much species as it is observed in natural communities. Available explanations answer this question in part only. Niche mechanisms can't explain the coexistence of species with strongly overlapping niches and "ecological twins". Numerous models, exploring the mechanisms that neutralize the competitive exclusion, argue that the species with certain characteristics can coexist, but do not answer the question why the characteristics of species are such that the observed in nature number of species can coexist. Furthermore, the vast majority of models, considering the dynamics of species in communities, address this issue at the individual species level, though interacting. Community as the specific system with its own laws of development is considered rare.
An approach that integrates processes at the levels of populations and communities may be helpful in this situation. The principle of optimal diversity of biosystems [3, 4], may serve as such an additional approach. This principle is based on the suggestion that the diversity of elements of a biosystem is related to the fundamental characteristics which define its viability (survival probability). The viability of a biosystem reaches its maximum at optimal value of its inner diversity. That is, the most viable populations have the optimal intrapopulation diversity and the most viable communities have the optimal species diversity.

\subsection{The model of Optimal Diversity without Niche Divergence}

To test this principle we have developed two models of optimal diversity at two interacting hierarchical levels populations and community.

The first model [3, 4] does not account for the phenomenon of niche divergence. A brief description of this model we repeat below. Environment is characterized by the intensity of the resources flow and by the degree of stability. At each moment of time, some value of an environmental parameter is realized, which can be interpreted as a characteristic of the most important resource (e.g., the size of prey) or as an environmental factor, providing its consumption (e.g., temperature). The dispersion of the distribution of its values defines the degree of environmental instability. Populations consist of various phenotypes. Phenotype characteristic is the ability of individuals to breed in a given environmental conditions. At each moment of time, the realized value of environmental parameter corresponds with a definite phenotype, for which the given environmental conditions are the most favorable. At each passing moment of time, the realized value of environmental parameter corresponds with a definite phenotype, for which the given environmental conditions are the most favorable. At this moment, a group of phenotypes breeds around it. The value of dispersion of distribution of breeding phenotypes can be interpreted as an index of the width of the zone of individual tolerance. The value of dispersion of distribution of their offspring serves as an index of diversity reproduced by the population at each step of its development. During the experiment, the population either dies or goes to the steady state with the definite numbers, level of resource consumption and phenotypic diversity.

The model community consists of populations sharing the same resource (the community of the one trophic status). This model considers that all the populations are identical in their parameters, i.e., the phenomena of dominance and niches separation are not considered. The optimization problem is solved on two hierarchical levels. The optimized parameter at the population level is the phenotypic diversity and at the community level the number of populations is (i.e., species). The criterion of optimality at both levels is the efficiency of resource use by biological systems. 
The results of mathematical modeling have showed the existence of optimal values of diversity which obtain maximum effectiveness of resource utilization at the population and community levels (the maximum total biomass under a certain quantity of the resource or the minimal consumption of resources at a certain total biomass). Optimal values of diversity depend on the intensity of resource flow and the instability of the environment. At that, dependence of the optimal values of intrapopulation and species diversity on the degree of environmental stability is of the opposite nature. Optimal species diversity increases in more stable and "rich" environments, while optimal intrapopulation diversity decreases in more stable environments and is independent of the intensity of resource flow.

\subsection{The Model of Optimal Diversity with Possibility of Niche Divergence}

In the second model populations have the ability to disperse on the axis of environmental parameter that is to separate niches. For simplicity then we will consider as an environmental parameter some characteristic of the resource, although, as in the first model can be considered any parameter, that allows populations to use the resource. The axis of resource parameter consists of a number of cells and forms a ring to avoid boundary effects. At every moment of time resource flows in some cells which are selected in random manner (Fig. 1). The degree of environmental stability is determined by the number of cells with resource at each step - the more the number of cells is the more stable the environment is. In this environment, there are several species, each of which consists of different phenotypes. As in the first model, the phenotypic feature - the ability to reproduce under certain environmental conditions, i.e. in a cell with resource with specific characteristics. Mechanisms of mortality, reproduction and distribution of phenotypes in the adjacent cells are similar to those in the first model. Initially, species are placed in each cell of the resource axis. After several generations the system passes to the steady state or all of the species die. The width of species phenotypic distribution is interpreted as the width of its ecological niche. Niches of species may overlap.

Values of resource parameter which are realized in the given moment of time

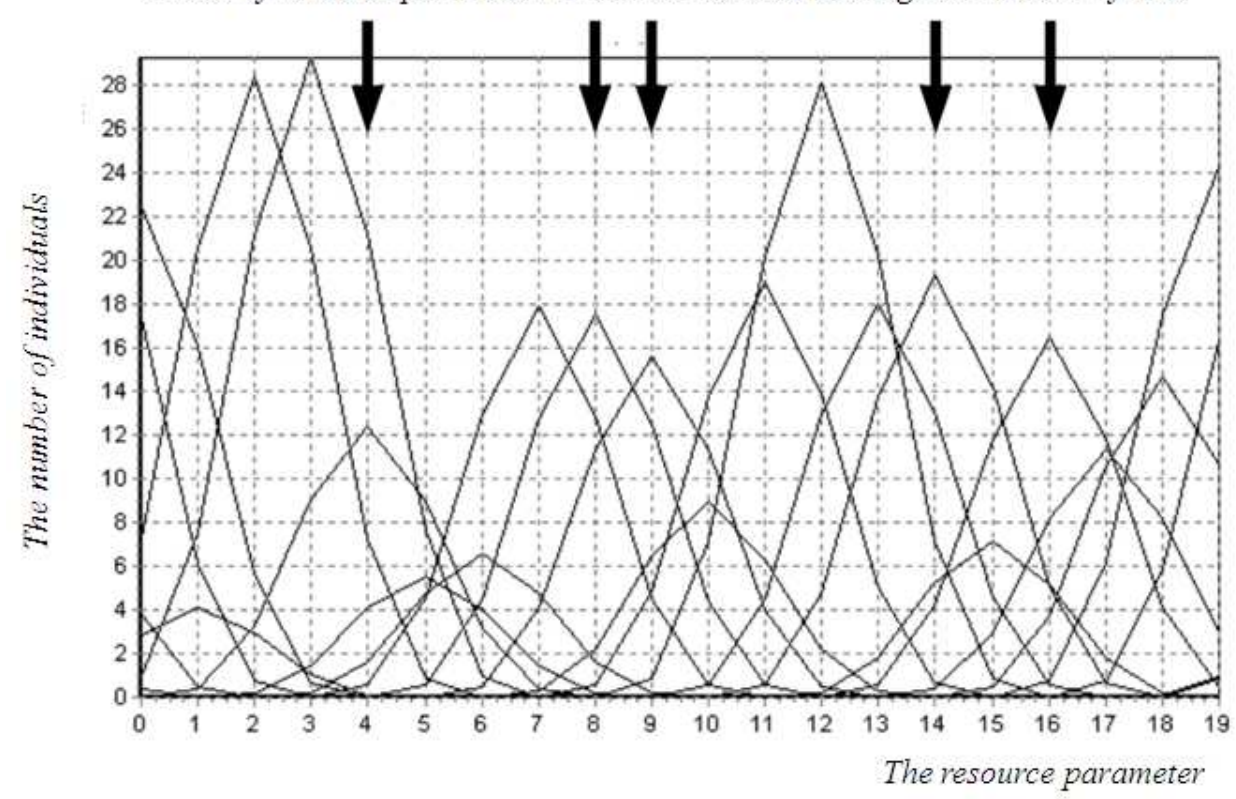

Figure 1. Distribution of species on the axis of the resource parameter at some moment of time. Each "bell" represents a population (option model with 20 cells).

A formal description of the model is as follows. Model community of one trophic level consists of $I$ populations. Each population consists of $J$ phenotypes, defined by their ability to reproduce when specific value of resource parameter is realized. $N_{i j}(t) \square$ is a number of individuals of $i$-th population and $j$-th phenotype at a time $t(i \in I, j \in J$, $t=1,2, \ldots)$. The set of recurrent balance equations has the following form:

$$
N_{i j}(t+1)=N_{i j}(t)-N_{i j}^{-}(t)+N_{i j}^{+}(t)
$$

$N_{i j}(t)$ is a number of individuals which died and $N_{i j}^{+}(t)$ is a number of individuals which born during the time interval $(t, t+1)$ in $j$-th phenotype of $i$-th population.

$$
\begin{gathered}
N_{i j}^{-}(t)=d_{i} N_{i j}(t) \\
N_{i j}^{+}(t)=r_{i j} \beta_{j j} N_{i j}(t)+r_{i j-1} \beta_{j-1 j} N_{i j-1}(t)+r_{i j+l} \beta_{j+l j} N_{i j+l}(t)
\end{gathered}
$$

In (2) and (3) $d_{i}$ is the mortality rate for the $i$-th species, $r_{i j}$ $\square$ the fertility rate for the relevant phenotype in $i$-th population, $\beta_{n m} \square$ the proportion of individuals born in the 
$m$-th phenotype and passed to the $n$-th phenotype. In accordance with the condition (3) individuals can only be born of this phenotype and phenotypes neighboring to it. Parameters $\beta_{n m}$ should be restricted with $\beta_{j j}+\beta_{j-1 j}+\beta_{j+1 j}=1$

The coefficients $r_{i j}(t)$ are defined in the following way:

$$
r_{i j}(t)=r_{\max }\left(1-K_{j}(t) / R_{j}(t)\right)(1-S(t) / R)
$$

where $r_{\max }$ - is the maximum allowable increase ratio; $K_{j}(t)=\sum_{i} N_{i j}(t)-$ is the sum of all individuals consuming the resource of the $j$-th value; $S(t)=\sum_{j} K_{j}(t) \square$ is a total number of individuals at time $t ; R_{j}(t)-$ is a random variable that determines the amount of the $j$-th resource, realized in time $t$; $\mathrm{R}=\sum_{j} R_{j}(t)=$ const $\square$ the amount of resource with a random distribution between the types of individuals.

The results of the model research, presented on Fig. 2,

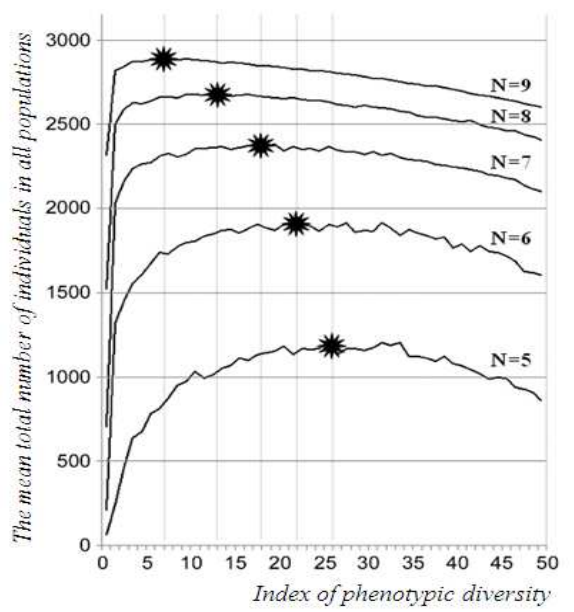

(a) show that the optimal values of intra-population diversity increases with the destabilization of the environment, while the total number of individuals in the community decrease (Fig. 2 a). The optimal number of species in the community reduces in the destabilization of the environment (Fig. 2 b).

Thus, qualitative character of changes in optimal values of diversity at the population and community levels depending on the degree of environmental stability is the same in two versions of the model: the optimal intrapopulation diversity (niche width) increases, and the optimal number of species reduces in less stable environments. Alternatively, the optimal number of species increases, and the width of niches decreases in more stable environments.

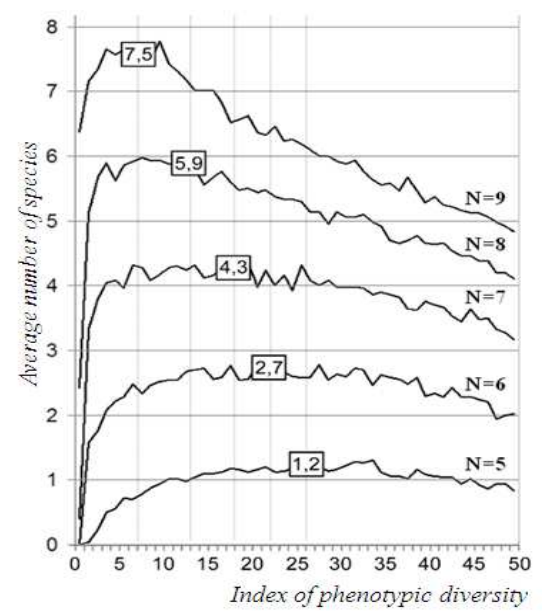

(b)

Figure 2. Increase the optimal phenotypic diversity (asterisks in Fig. a) and decrease the optimal number of species (numbers in frames in Fig. b) in the destabilization of the environment. $N$ is number of cells with the resource influx which corresponds with stability of environment.

\subsection{Combined Effect of Optimization, Niche and Neutral Mechanisms}

Based on the fact that the optimal values of diversity occur in both models, we assume that the optimization of diversity can be the specific mechanism of diversity formation. Thus, at least three groups of mechanisms can take part in determining of the number of species in the community: niche, neutral and optimization mechanisms (Fig. 3). Optimal values of species and intrapopulation diversity are determined by the "richness" and stability of environment and the evolutionary level biota [3, 4]. The feasibility of these optimal values is determined by the regional species pool during the interaction of species through niche and neutral mechanisms.

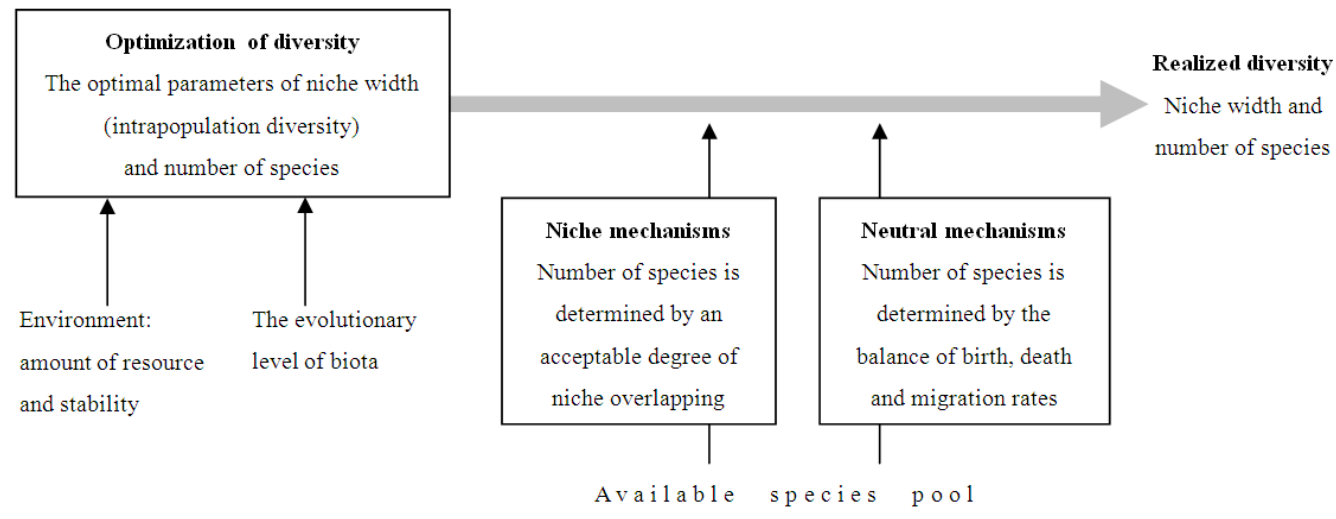

Figure 3. Three groups of mechanisms, forming species diversity of communities 
Optimization, niche and neutral mechanisms do not contradict each other, it is only necessary to determine the ranges of their impacts and their ratio in different conditions. Obviously, in different habitats and conditions and at different successional stages, and depending on the characteristics of species in a regional pool (e.g., the ratio of $\mathrm{r}$ - and K-strategists), different mechanisms may have different importance.

In order to determine the relation of these three groups of mechanisms we have considered the simplest thought experiment, ignoring the environment heterogeneity, community development and species characters. In axes of resource amount and environmental stability consider a stationary state of the community of species with similar characteristics, of which can only be changed niche width and resource consumption. For clarity, we divide the space conditions in the axes of "resource $\square$ stability" into cells (Fig. 4). Resource parameter range $(D)$ in all cells is identical, for example the size of feed particles ranges anywhere from 1 to $5 \mathrm{~mm}$ (in Fig. 4 this range corresponds to the total width of niches in each cell). However, the stability of the resource influx is different: in stable conditions (left column of cells) the population receives particles of different sizes at each step of the development, in unstable conditions (right column) $\square$ just one size, and every time $\square$ different. The cells include populations with the optimal niche width $\left(D^{*}\right)$, except for cell 3 , where the niche width is wider than the optimum (see below). The volume of incoming resource $(R)$ increases from the lower to the upper row of cells, $R^{*} \square$ the resource volume required for a population with an optimal niche width, $R_{\text {min }}$ - the minimum resource volume on which a population can exist.

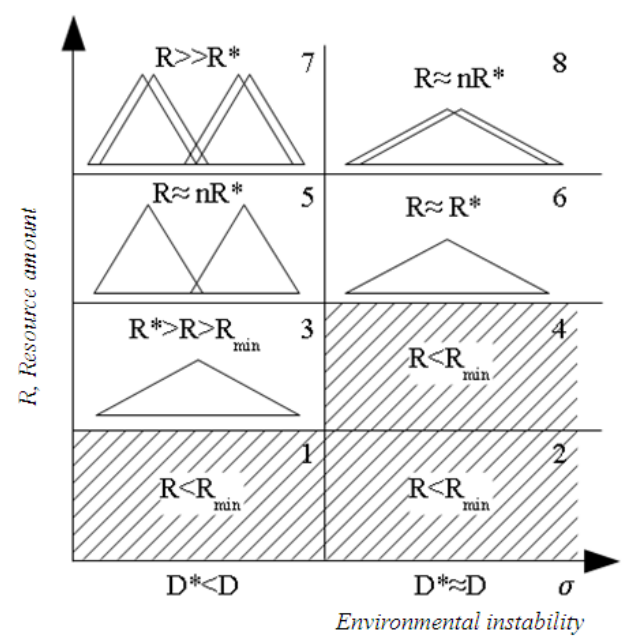

Figure 4. Width and number of niches according to the ratio of available resource and environmental instability. $R$ - the amount of resources available in each cell; $R^{*} \square$ the amount of resource, which is necessary for the population with an optimal niche width, $R_{\min }-$ the minimal amount of resource which is necessary for population existence; $D$ - the range of resource parameter value, $D^{*} \square$ optimal niche width. Triangles represent schematically the phenotypic distribution of populations, i.e. the width of the niches.
In accordance with the principle of optimal diversity the optimal niche width $D^{*}$ is determined by a degree of the environmental instability, and is wider in unstable environment. The optimal number of species is determined by a degree of the environmental instability and available resources volume, or in other words, by how many species with the optimum niche width for the given conditions can exist on the incoming resource.

No one species can exist at very low rates of the resource inflow ( $R<R_{\min }$, cells 1 and 2 ) even in stable environment. With an increase in resource (cells 3 and 4) it is enough only for the existence of one species in a stable environment, but in such a poor environment this species have to occupy a larger niche than the optimal one to obtain enough of resources $\left(R^{*}<R<R_{\min }\right.$, the cell 3). In case of an even greater resource inflow several species can coexist (two are shown) in a stable environment $\left(R \approx n R^{*}\right.$, cell 5) and one $\square$ in an unstable $\left(R \approx R^{*}\right.$, cell 6$)$, since the existence in an unstable environment implies greater resource demand. Lastly, if resource abundance is even greatly, many species can exist in stable conditions $(R>>$ $R^{*}$, cell 7) and a small number of species $\square$ in the unstable (cell 8).

Why the number of species in our scheme increases with an increase of resource influx instead of the number of one winning species increase? If we consider an individual species level, it seems illogical. But when consider the community level, the picture is quite different. It is shown that the species diversity in the range from 1 to the natural number of species allows more efficient using of the environmental resources due to effects of complementarity and asynchrony, when the species differences in resource use, and asynchronous changes in their numbers in response to environmental fluctuations provide greater and more stable total productivity of the community $[44,45]$. However, our models let us suggest that increasing of the community effectiveness in this range of species numbers can occur without niche divergence between species. This is evidenced by the emergence of the optimal values of the number of species more than " 1 " without niches divergence. In either case, the community with one species, that will displace all others, will use the resources influx less efficient and, eventually, will be displaced by other more optimal community through mechanisms similar to shift between succession stages.

The optimal values of species number and width of their niches are modified by niche and neutral mechanisms. Their relative importance depends on environmental conditions. If the resource is very abundant (cell 7) several species with similar ecological characteristics may exist in the same niche and species composition will be primarily determined by neutral mechanisms. It can be compared with the conditions of tropical forests as an example of which the neutral theory was developed. The similar patterns can also be expected in a very stable environment, with a medium intensity of resource influx. The results of 
our modeling show that the niche overlap index for the same resource increases while the environment is stabilizing (Fig. 5a). One would expect that the degree of niche overlapping will decrease with environmental

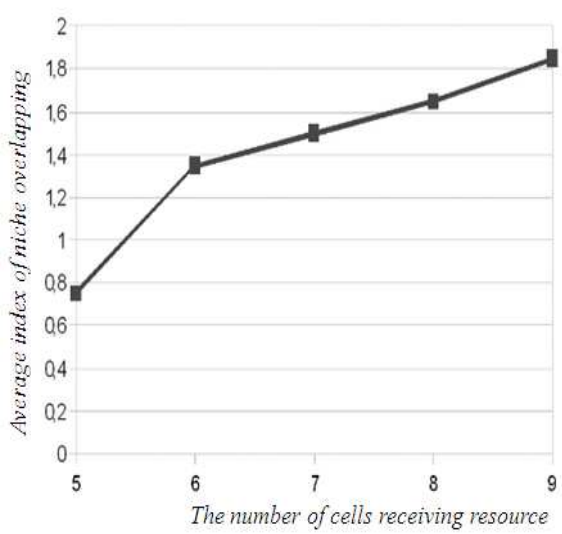

(a) stabilization, since the width of niches contracts. However, the number of species increases (Fig. 5b), and the effect of the species number increasing affects the niche overlap value stronger than contracting of the niches do.

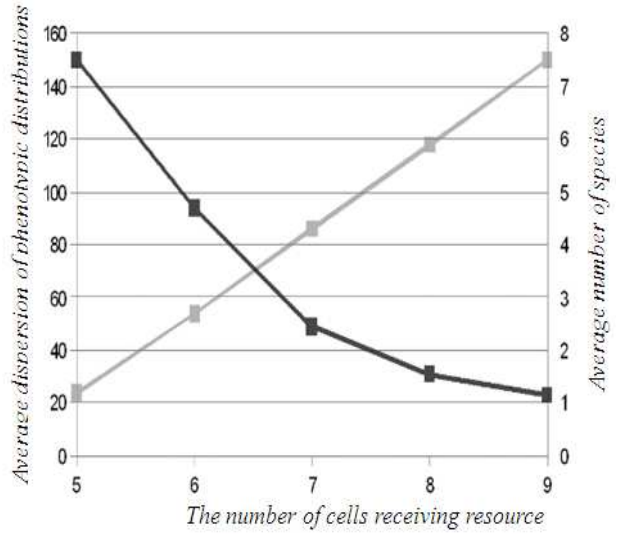

(b)

Figure 5. Increase of niches overlapping (a), increase of the optimal values of species number and decrease of niche width (b) in more stable environments. The proxy of the environmental stability is the number of cells receiving the resource at each step of the modeling. The proxy of niche width is dispersion of phenotypic distributions.

If the amount of resource is too small (cell 3), the niche separation does not occur, since only one species can survive in the cell in these harsh environment. These conditions can be collated with extreme habitats in which species composition is determined primarily by selection of species, resistant to abiotic factors ("abiotic filters"). For example, S. Sheremetyev and Y. Gamaley [46] showed that in arid conditions plant communities consist of stress-tolerant species with similar water regime and species composition is determined primarily by ecotopic selection, not by competitive relations. In areas with wetter conditions the ecotopic selection becomes weaker and the competitive relations grow stronger resulting in divergence of species niches. Therefore, these communities are represented by the species with different characteristics of water exchange.

The niche divergence between species can occur in the most explicit form in cell 5, where the optimum width of the niche is less than an accessible range of the resource parameter $\left(D^{*}<D\right)$ and the amount of the resource is enough for a small number of species $\left(R=n R^{*}\right)$. Competition can be a major factor in shaping the community, if $D / D^{*} \approx R / R^{*} \square$ that is, the number of niches with optimal width, which divide the available range of the resource parameter and the number of species that may exist on the available quantity of the resource, are approximately equal.

Thus, the predominance of a particular type of mechanisms of community structure forming in our scheme is determined not only by "richness" and stability of the environment but by the ratio of these characteristics with regard to the requirements and adaptive capacities of species, indicated by a ratio of $D / D^{*}$ and $R / R^{*}$ (Fig.6).

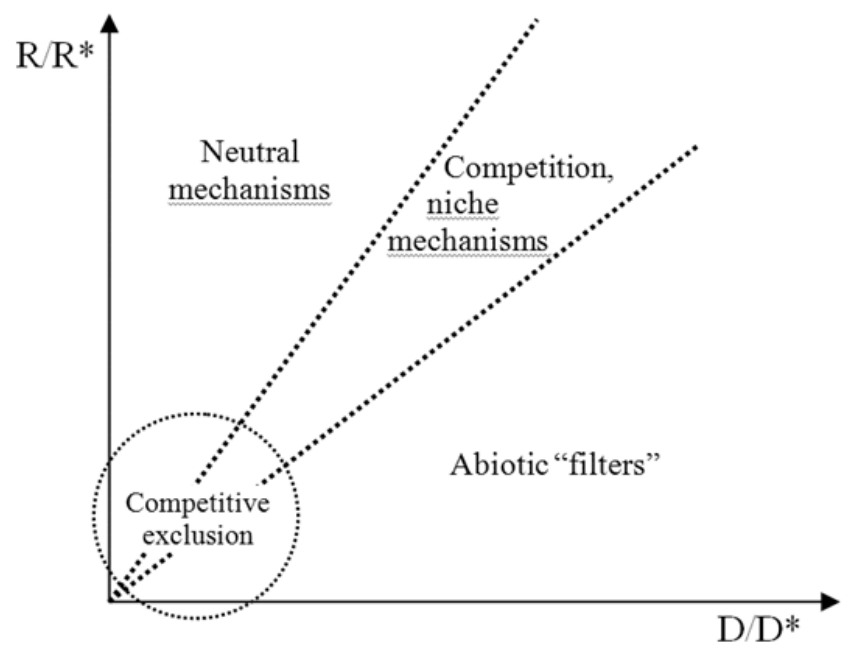

Figure 6. The main mechanisms that shape diversity. $D / D^{*}-$ is the number of niches with optimal width that may exist on the available range of resource parameter; $R / R^{*}$ - is the number of species with optimal niches that may exist on the available amount of resource

Above the zone of approximate equality of $D / D^{*} \approx R / R^{*}$ where we expect the most obvious manifestation of the competition, a powerful flow of resource allows the existence of many more species than the optimal number of available niches and predominance of neutral mechanisms in the formation of community. Below the zone $D / D^{*} \approx$ $R / R^{*}$, on the contrary, the number of species, which can live on the given resource, significantly less than the number of optimal niches, which is why some of them may remain empty. Therefore, competition in this area is also likely to be weak, and the major role in shaping the community will play an "abiotic filters". Examples of competitive exclusion may be probably found in the zone, 
adjacent to zero, where the volume and range of resources are enough only for a very limited number of species.

\section{Conclusion}

Basing on the principle of optimal diversity, the following scheme of different mechanisms of diversity formation can be proposed:

$1 \square$ number of species and their niche width are primarily determined by diversity optimization, in accordance with volume of the available resources and the degree of the environmental stability;

$2 \square$ during formation of the species composition the modification of diversity levels occurs through neutral, niche and abiotic mechanisms the relative importance of which depends on the ratio of environmental "richness" and stability:

$\square$ neutral mechanisms work primarily in very "rich" or very stable environments;

$\square$ niche mechanisms work primarily in the environments with medium values of the "richness" and stability, rather when the number of niches with optimal width, which divide the available range of the resource parameter and the number of species that may exist on the available quantity of the resource, are approximately equal;

"abiotic filters" work primarily in poor, severe, or extremely unstable environments.

Therefore, answering the question posed in the title, we can assume that niche divergence is not a necessary condition for the formation of community species diversity. The number of species in the community and the width of their niches are determined by diversity optimization, when competition and divergence of species niches modify the structure depending on environmental conditions, the regional species pools characteristics, successional stages, etc. In very "reach" and stable environment when niche mechanisms are of little importance, the observed differences in species niches may be the result of a random combination of various factors. For example, it has been shown that small differences in niches of previously mentioned grass and moor frogs are determined not by competition in the modern community, but by the specific history of speciation [14, 47].

A variety of factors influencing the species formation is so great that the likelihood of emergence of completely ecologically identical species is almost zero $\square$ particular ecological differences will always be present. In a significant range of conditions they are not the major factor in forming of community structure, but only $\square$ optional one which, however, may give some advantages to the community due to the effects of complementarity and asynchrony (see above). A similar assumption can be made about sympatric speciation in "rich" and stable environment. Thus, N. Orlov [48] describes the formation of a kind of "communities" of sympatric sibling species of tropical frogs which differ only in minor behavioral and ecological aspects. What may be a major factor in the formation of such complexes $\square$ the niche divergence or the diversity optimization? In our view, it seems unlikely that minor differences in ecological niches may play a role of energetically significant driving force of speciation process. Perhaps the major factor here may be the optimization of inter- and intraspecific diversity. In a stable and "rich" environment the optimal number of species increases and the optimal niche width narrows. This is what is observed: one species splits into several slightly more specialized species.

Of course, all this reasoning expressed in the article are only preliminary hypothesis requiring further investigation. But we hope that the analysis of the possible optimization mechanisms at the population and community levels may be useful in understanding the ways of the biodiversity formation.

\section{Acknowledgements}

The authors are grateful to A. Severtsov for constructive discussion of the article and valuable additions that have been used in the preparation of the publication.

The research was performed under the Program of the Presidium of the Russian Academy of Sciences "Wildlife: Current Status and Problems of Development”.

\section{References}

[1] Millennium Ecosystem Assessment. Ecosystems and Human Wellbeing: Synthesis. Island Press, Washington, DC. 2005.

[2] J. M. Chase and M.A. Leibold. Ecological Niches: linking classical and contemporary approaches. Chicago University Press, 2003, 212 p.

[3] G. M. Aleshchenko and E. N. Bukvareva, "Two-Level Hierarchical Model of Optimal Biological Diversity," Biology Bulletin, vol. 37, № 1, pp. 1-9, 2010.

[4] G. M. Aleshchenko and E. N. Bukvareva, "The Principle of Optimal Biodiversity and Ecosystem Functioning," International Journal of Ecosystem, vol. 2, № 4, pp. 78-87, 2012.

[5] M. W. Palmer, "Variation in species richness: towards a unification of hypotheses," Folia Geobot. Phytotaxon., vol. 29, № 4, pp. 511-530, 1994.

[6] R. M. May, R. H. MacArthur, "Niche overlap as a function of environmental variability," Proc. Nat. Acad. Sci. USA, vol. 69, № 5, pp. 1109-1113, 1972.

[7] R. M. May, Stability and complexity in model ecosystems, Princeton Univ. Press, 235 p. 1973.

[8] P. Szabo and G. Meszéna, "Limiting Similarity Revisited," Oikos, vol. 112, № 3, pp. 612-619, 2005.

[9] 9 R. Petersen, "The Paradox of the Plankton: An Equilibrium Hypothesis,” The American Naturalist, vol. 109, № 965, pp. 35-49, 1975.

[10] D. Tilman 1982. Resource competition and community structure. Princeton University Press, Princeton, New Jersey. 
296 p.

[11] J. H. Connell, "Diversity in rain forests and coral reefs," Science, vol. 199, № 4335, pp. 1302-1309, 1978.

[12] S. P. Hubbell, "Neutral theory in community ecology and the hypothesis of functional equivalence," Functional Ecology, vol. 19, № 1, pp. 166-172, 2005.

[13] J. S. Clark, "Individuals and the variation needed for high species diversity in forest trees," Science, vol. 327, № 5969, pp. 1129-1132, 2010.

[14] A. S. Severtsov, S. M. Lyapkov and G. S. Surova, "The ratio of ecological niches grass (Rana temporaria L.) and the moor (Rana arvalis Nilss.) frogs (Anura, Amphibia)," Zhurnal obschey biologii, vol. 59, № 3, pp. 279-301, 1998 (in Russian).

[15] B. Herault, A. Bornet and M. Tremolieres, "Redundancy and niche differentiation among the European invasive Elodea species,” Biol. Invasions, vol. 10, № 7, pp. 1099-1107, 2008.

[16] M. Pfenninger and K. Schwenk, "Cryptic animal species are homogeneously distributed among taxa and biogeographical regions," BMC Evol. Biol., vol. 7, pp. 121-126, 2007.

[17] D. Bickford, D. J. Lohman, N. S. Sodhi, P. K. L. Ng, R. Meier $\mathrm{K}$. Winker at al., "Cryptic species as a window on diversity and conservation," TRENDS in Ecology and Evolution, vol. 22, № 3, pp. 148 - 155, 2006 .

[18] C. P. Blair, W. G. Abrahamson, J. A. Jackman and L. Tyrrell, "Cryptic speciation and host-race formation in a purportedly generalist tumbling flower beetle,” Evolution, vol. 59, № 2, pp. 304-316, 2005.

[19] J. O. Stireman III, J. D. Nason and S. B. Heard, "Host-associated genetic differentiation in phytophagous insects: general phenomenon or isolated exceptions? Evidence from a goldenrod-insect community," Evolution, vol. 59, № 12, pp. 2573-2587, 2005.

[20] P. D. N. Hebert, E. H. Penton, J. M. Burns, D. H. Janzen and W. Hallwachs, "Ten species in one: DNA barcoding reveals cryptic species in the neotropical skipper butterfly Astraptes fulgerator,” Proc. Natl. Acad. Sci. U.S.A, vol. 101, № 41, pp. 14812-14817, 2004.

[21] M. Kankare, S. Van Nouhuys and I. Hanski, "Genetic divergence among host-specific cryptic species in Cotesia melitaearum aggregate (Hymenoptera: Braconidae), parasitoids of checkerspot ," Ann. Entomol. Soc. Am., vol. 98, № 3, pp. 382-394, 2005.

[22] M. A. Smith, N. E. Woodley, D. H. Janzen, W. Hallwachs and P. D. N. Hebert, "DNA barcodes reveal cryptic host-specificity within the presumed polyphagous members of a genus of parasitoid flies (Diptera: Tachinidae)," Proc. Natl. Acad. Sci. U. S. A, vol. 103, № 10, pp. 3657-3662, 2006.

[23] D. Molbo, C. A. Machado, J. G. Sevenster, L. Keller and E. A. Herre, "Cryptic species of fig-pollinating wasps: implications for the evolution of the fig-wasp mutualism, sex allocation, and precision of adaptation," Proc. Natl Acad. Sci. USA, vol. 100, № 10, pp. 5867-5872, 2003.

[24] J. B. Wilson, "Mechanisms of species coexistence: twelve explanations for Hutchinson's "paradox of the nplancton": evidence from New Sealand plant communities," New
Zealand Journal of Ecology, vol. 13, № 1, pp.17-42, 1990.

[25] P. L. Chesson, "Mechanisms of maintenance of species diversity," Annual Review of Ecology and Systematics, vol. 31, pp. 343-366, 2000.

[26] S. J. Wright, "Plant diversity in tropical forests: a review of mechanisms of species coexistenc," Oecologia, vol. 130, № 1 , pp. 1-14, 2002.

[27] S. Barot, "Mechanisms promoting plant coexistence: can all the proposed processes be reconciled?" Oikos, vol. 106, № 1, pp. 185-192, 2004.

[28] J. M. Chase, "Towards a really unified theory for metacommunities," Functional Ecology, vol. 19, № 1, pp. 182-186, 2005

[29] S. P. Hubbell, The Unified Neutral Theory of Biodiversity and Biogeography, Princeton University Press, Princeton, NJ, 448 p., 2001.

[30] G. Bell, "The distribution of abundance in neutral communities,” The American Naturalist, vol. 155, № 5, pp. 606-617, 2000

[31] G. Bell, "Neutral macroecology,” Science, vol. 293, № 5539, pp. 2413-2418, 2001

[32] C. P. Doncaster, "Ecological equivalence: a realistic assumption for niche theory as a testable alternative to neutral theory," PLoS ONE, vol. 4, № 10, 8 p., 2009.

[33] J. S. Clark, "Beyond neutral science // Trends in Ecology and Evolution,” V. 24, № 1, pp. 8-15, 2008

[34] K. J. Gaston and S. L. Chown, "Neutrality and the niche," Functional Ecology, vol. 19, № 1, pp. 1-6, 2005.

[35] M. Holyoak and M. Loreau, "Reconciling empirical ecology with neutral community models," Ecology, vol. 87, № 6, pp. 1370-1377, 2006.

[36] I. Volkov, J. R. Banavar, S. P. Hubbell and A. Maritan, "Patterns of relative species abundance in rainforests and coral reefs," Nature, vol. 450, № 7166, pp. 45-49, 2007.

[37] G. Bell, M. J. Lechowicz and M. J. Waterway, "The comparative evidence relating to functional and neutral interprtetations of biological communities," Ecology, vol. 87, № 6, pp. 1378-1386, 2006.

[38] B. J. McGill, B. A. Maurer and M. D. Weiser, "Empirical evaluation of neutral theory," Ecology, vol. 87, № 6, pp. $1411-1423,2006$

[39] P. B. Adler, J. Hillerislambers and J. M. Levine, "A niche for neutrality,” Ecology Letters, vol. 10, № 2, pp. 95-104, 2007.

[40] J. Chave, H. C. Muller-Landau and S. A. Levin, "Comparing classical community models: theoretical consequences for patterns of diversity,” The American Naturalist, vol. 159, № 1, pp. 1-23, 2002.

[41] D. Gravel, C. D. Canham, M. Beaudet and C. Messier, "Reconciling niche and neutrality: the continuum hypothesis," Ecology Letters, vol. 9, № 4, pp. 399-409, 2006.

[42] B. Herault, "Reconciling niche and neutrality through the Emergent Group approach," Perspectives in Plant Ecology, Evolution and Systematics, vol. 9, № 2, pp. 71-78, 2007. 
[43] M. Scheffer and E.H. van Nes, "Self-organized similarity, the evolutionary emergence of groups of similar species," Proc. Natl. Acad. Sci. USA, vol. 103, № 16, pp. 6230-6235, 2006.

[44] E. M. Spehn, A. Hector, J. Joshi, M. Scherer-Lorenzen, B. Schmid, E. Bazeley-White et al., "Ecosystem effects of biodiversity manipulations in European grasslands," Ecological Monographs, vol. 75, № 1, pp. 37-63, 2005.

[45] A. Hector, Y. Hautier, P. Saner, L. Wacker, R. Bagchi, J. Joshi et al., "General stabilizing effects of plant diversity on grassland productivity through population asynchrony and overyielding," Ecology, vol. 91, № 8, pp. 2213-2220, 2010.
[46] S. N. Sheremet'ev and Y. Gamaley, "Trends in ecological evolution of herbs," Zhurnal obschey biologii, vol. 70, № 6, pp. 459-483, 2009 (in Russian).

[47] A.S. Severtsov, "Microevolution and phylocoenogenesis," Evolutionary Biology. Proceedings of the conference "The problem of species and speciation," vol.1. Tomsk: Tomsk State University, pp. 89-105, 2001 (in Russian).

[48] N. L. Orlov, "Vietnam cryptic species of amphibians and their importance for the evaluation of the taxonomic diversity," Uspehi sovremennoy biologii, vol. 127, № 6, pp. 612-621, 2007 (in Russian). 